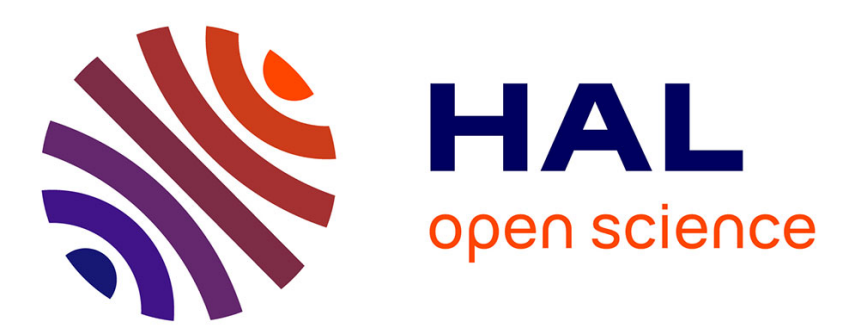

\title{
TIC, tac, tic, tac... Du temps traqué au travail contrôlé : le cas du transport routier de marchandises
}

\author{
Anne-France Kogan
}

\section{To cite this version:}

Anne-France Kogan. TIC, tac, tic, tac... Du temps traqué au travail contrôlé: le cas du transport routier de marchandises. Tic\&société, 2016, Contrôle social, surveillance et dispositifs numériques, 10 (1). hal-01416645

\section{HAL Id: hal-01416645 \\ https://hal.science/hal-01416645}

Submitted on 14 Dec 2016

HAL is a multi-disciplinary open access archive for the deposit and dissemination of scientific research documents, whether they are published or not. The documents may come from teaching and research institutions in France or abroad, or from public or private research centers.
L'archive ouverte pluridisciplinaire HAL, est destinée au dépôt et à la diffusion de documents scientifiques de niveau recherche, publiés ou non, émanant des établissements d'enseignement et de recherche français ou étrangers, des laboratoires publics ou privés. 


\section{tic\&société}

Vol. 10, $\mathrm{N}^{\circ} 1 \mid 2016$

Contrôle social, surveillance et dispositifs numériques

\section{TIC, tac, tic, tac... Du temps traqué au travail contrôlé : le cas du transport routier de marchandises}

Anne-France KOGAN

revues.org Éditeur

Association ARTIC

Édition électronique

URL : http://ticetsociete.revues.org/1998

Référence électronique

Anne-France KOGAN, «TIC, tac, tic, tac... Du temps traqué au travail contrôlé : le cas du transport routier de marchandises », tic\&société [En ligne], Vol. 10, № 1 | 1er semestre 2016, mis en ligne le 15 octobre 2016, consulté le 06 novembre 2016. URL : http://ticetsociete.revues.org/1998

Ce document a été généré automatiquement le 6 novembre 2016

Licence Creative Commons 


\title{
TIC, tac, tic, tac... Du temps traqué au travail contrôlé : le cas du transport routier de marchandises
}

\author{
Anne-France KOGAN
}

\section{Introduction}

1 La complexité et la diversité des transformations associées aux TIC conduisent à réfuter toute hypothèse d'un impact univoque de la technique sur l'organisation (Gollac et al., 1999). Des recherches plus qualitatives sur l'articulation entre travail et TIC tentent de comprendre comment ces techniques numériques s'immiscent dans l'organisation et comment elles transforment le travail. Elles mettent fin à toute idée de déterminisme technique et soulignent que l'on ne peut comprendre ces transformations sans considérer, d'une part, les situations sociales préexistantes et, d'autre part, les apprentissages individuels et collectifs des acteurs qui se saisissent de ces technologies dans le travail.

2 En interrogeant plus particulièrement le lien entre TIC et conditions de travail, les chercheurs s'accordent pour avancer que ce lien n'est pas aussi univoque que l'on peut penser, car il reste médié par l'organisation. L'usage des TIC n'est pas le seul déterminant des conditions de travail, d'autres facteurs interviennent (Klein et Ratier, 2012). Le cas des centres d'appel, qui reste exemplaire des mutations du travail associées aux usages des technologies numériques, montre que la nature des contrats qui lient les donneurs d'ordres aux centres d'appel ou la complexité des savoir-faire qu'exige la réponse aux appels entrants influent sur les conditions de travail. Le niveau de technicité des réponses et des contenus des échanges informationnels distingue en grande partie le degré de pression du flux des appels et donc les conditions de travail. Autrement dit, plus la compétence est élevée, " plus la pression se réduit, car, d’une part, la qualité du service requiert du temps et, d'autre part, la rareté de la main-d'œuvre qualifiée acceptant de 
travailler dans les CAT conduit à la ménager relativement » (Durand, 2004, p. 168). Par ailleurs, le secteur dans lequel s'opère ce service intervient également sur la pression du flux et le stress qu'il génère : ainsi, dans le secteur de la téléphonie sociale, la logique productive s'efface derrière celle de l'aide. Les téléopérateurs, qu'ils soient salariés ou bénévoles, sont donc beaucoup moins soumis à cette pression (Kogan, 2009).

Néanmoins, avec toutes les informations produites sur le travail via les TIC, les relations entre autonomie et contrôle se complexifient, dépassant les antagonismes d'hier. Les TIC contribuent au renforcement du contrôle, mais ne peuvent pas être considérées comme en étant la seule cause. Celui-ci découle, en réalité, de la mise en œuvre de normes de production ou de qualité ainsi que des intentions managériales qui les orientent. L'efficacité des TIC dans ce domaine va cependant multiplier les effets du contrôle, parfois au-delà des besoins et des intentions.

Deux grandes modalités de contrôle sont néanmoins associées aux TIC: la mise en visibilité de l'activité et l'impératif temporel.

5 La visibilité des individus et des collectifs de travail s'appuie de plus en plus sur des dispositifs numériques (Andonova et Vacher, 2013). Ceux-ci produisent de nombreuses traces et favorisent un contrôle indirect sur les résultats de leurs activités. Dès lors, les salariés bénéficient d'une autonomie plus importante dans l'organisation et la réalisation opérationnelle de leur travail. Les TIC participent alors à la construction d'une «autonomie encadrée »: les salariés doivent arbitrer en permanence entre différents choix pour obtenir un résultat « sous contrôle » (Gheorghiu et Moatty, 2005).

La diffusion des TIC va de pair avec la montée des outils de gestion, qui s'impose comme une nouvelle forme de gouvernance des entreprises. Le management intermédiaire consacre de plus en plus de son temps à réaliser des tableaux de bord, des graphiques pour visualiser, contrôler et chasser le moindre temps mort et tout gaspillage. Cela a permis d'augmenter la productivité par une production en juste-à-temps, une qualité intégrée et une standardisation des tâches. Dès lors, instantanéité, immédiateté, simultanéité et visibilité sont des maîtres mots qui dictent le quotidien des entreprises, des équipes de travail et des salariés (Aubert, 2003). Cet avènement de l'hypermodernité renvoie à un rapport au temps toujours plus court, avec la volonté de maîtriser le temps, de le dominer, aboutissement ultime de la logique capitaliste. Cette domination du temps par l'individu a pris, à notre époque, deux visages : celui de l'instantanéité, d'une part, permise par les nouvelles technologies qui donnent à l'individu le sentiment de pouvoir abolir le temps, et celui de l'urgence, d'autre part, qui constitue une nouvelle forme de contrainte, voire de violence (Aubert, 2006). Cette instauration de l'urgence comme idéologie générale des sociétés contemporaines est déjà présente dans le fordisme et le toyotisme où l'injonction à gagner du temps, associée à une menace (de perdre son emploi, d'être sous-évalué, etc.), constitue le fil directeur de ces concepts d'organisation du travail: alors que le salarié vend un temps de travail à son employeur, celui-ci l'exploite en le densifiant, c'est-à-dire en introduisant de plus en plus d'actions dans le même temps, éventuellement raccourci. Cela vaut pour les ouvriers, comme pour les cadres (Bouton, 2013).

7 Le déploiement des TIC est fortement articulé aux questions temporelles (Vendramin, 2006). Elles s'inscrivent en cela dans l'histoire longue de l'écriture et de ses supports qui ont soutenu le processus de distanciation « spatio-temporelle » par dissociation du temps et de l'espace et de leur redistribution. Les technologies numériques ont donc fort à voir avec la vitesse, la réduction des «temps morts » et des délais, comme si elles pouvaient 
permettre de s'abstraire du temps, d'offrir le don d'ubiquité. Autrement dit, «il existe donc bien une dimension cachée de la révolution des communications qui affecte la durée, le temps vécu de nos sociétés » (Virilio, 1995, p. 36).

Cette idéologie de l'immédiateté et de l'urgence permet de mettre en œuvre des modalités de contrôle, justifiées non pas par la nécessité du contrôle lui-même mais par l'impératif économique.

9 Nous prendrons pour exemple le cas du secteur du transport routier de marchandises (TRM). En se situant sur un maillon de la chaîne de la production ou en fin de chaîne en distribuant des produits pour la consommation, ce secteur est exemplaire dans sa capacité à avoir intégré les contraintes temporelles malgré la contrainte incontournable $\mathrm{du}$ franchissement des espaces physiques. En effet, en mobilisant l'argument temporel comme seul argument d'efficacité, l'organisation du fret a construit à la fois une société disciplinaire (la discipline du flux auquel on se soumet) et une société de contrôle (la prolifération des traces numériques a des effets incitateurs sur les comportements). Avant de développer cette hypothèse, revenons sur les grandes caractéristiques du secteur du TRM et de ses évolutions.

10 Cet article s'appuie sur une recherche menée en 2012 pour laquelle une trentaine d'entretiens semi-directifs ont été réalisés dans dix entreprises du TRM dans le cadre d'un CEP Transports ${ }^{1}$. Pour chaque entreprise, nous avons rencontré le directeur en charge des questions RH et deux ou trois salariés, des conducteurs $\mathrm{VL}$ ou $\mathrm{PL}^{2}$ pour la plupart. Pour aborder la question des pratiques de communication, qu'elles soient privées ou professionnelles, une dizaine d'entretiens supplémentaires ont été réalisés dans des cafétérias d'autoroutes et de restaurants routiers.

\section{Le secteur du TRM}

11 Le transport de marchandises désigne la phase de déplacement physique de la marchandise entre deux points séparés géographiquement et ce, sans transformation. Pour Bernardet (1999), le développement du TRM est «le reflet des transformations structurelles de notre économie »; ses évolutions incorporent donc celles du monde productif.

12 Premièrement, une transformation de la nature des productions et des produits a été observée. Le poids de l'industrie lourde et de ses productions de produits pondéreux a considérablement diminué et ainsi laissé la place à des produits à forte valeur ajoutée, impliquant de plus faibles quantités à transporter à l'avantage du TRM sur les autres modes de transport (fluvial, ferré).

13 Deuxièmement, l'éclatement du système de production et de distribution que l'on a pu observer depuis le début des années 1980 a eu pour conséquence de placer en son cœur le système de transport. C'est son efficacité à livrer rapidement plusieurs points qui a permis au système productif dans la sélection de ses modes d'approvisionnement de n'accorder qu'un choix relativement secondaire à leur localisation et ainsi de pouvoir faire venir, si nécessaire, les pièces et sous-ensembles d'une région éloignée. Cette complexité croissante du système productif a donc eu pour conséquence directe d'accroître le volume des flux. Cette transformation a particulièrement complexifié l'organisation des transports. 

chronotachygraphe, un ordinateur de bord, un GPS et un, voire deux téléphones portables.

Les technologies embarquées

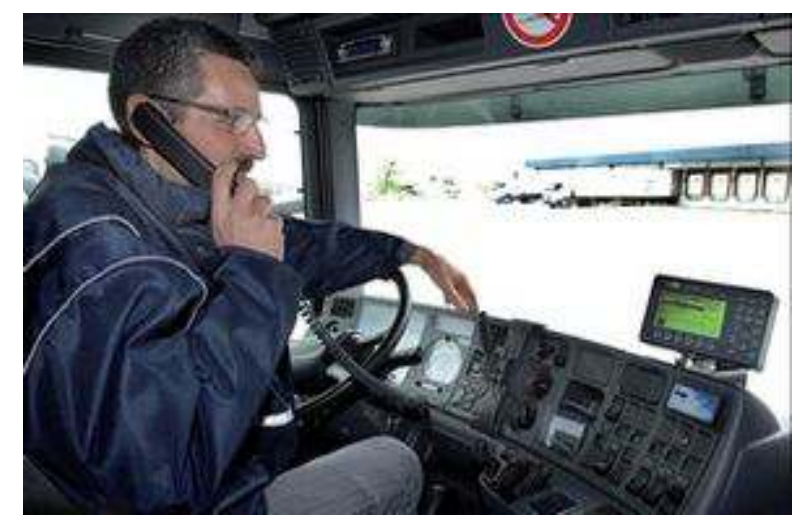

Troisième facteur: le développement du juste-à-temps. La prise de conscience de l'importance des coûts de stockage (en termes de surface, de main-d'œuvre et de frais de gestion, notamment) s'inscrit dans un phénomène plus large de développement et d'imposition d'une logique d'optimisation de la productivité globale des facteurs (équipement, travail, matière première et donc stocks) devant faire face à une demande plus volatile et à un marché très concurrentiel. Le juste-à-temps implique de fait la compression des stocks et, par là, la multiplication des flux d'approvisionnements. Pour les transporteurs, confrontés à une logique de rentabilité, cette multiplication des points de livraison s'est accompagnée d'une augmentation de colis et d'une diminution des volumes à livrer chez chaque client.

Quatrième facteur : l'augmentation des contraintes réglementaires et particulièrement le contrôle du temps de travail des conducteurs routiers. Celle-ci a eu pour conséquence directe l'augmentation des tarifs et finalement l'externalisation progressive de l'activité transport des entreprises.

Un cinquième facteur, plus récent, est l'augmentation des achats sur Internet qui se traduit par une hyperfragmentation de la distribution.

Du fait de l'ensemble de ces évolutions, le métier de transporteur se complexifie. Il donne une place centrale au travail d'organisation et de suivi des tournées de livraisons et de rechargements. Cela se traduit au recours croissant à l'externalisation. En 1995, les statistiques ${ }^{3}$ révèlent un décrochage des transports en compte propre (l'entreprise transporte ses propres produits) au profit du transport pour compte d'autrui (des entreprises dédiées à l'activité du TRM). Ces entreprises investissent dans des systèmes informatiques d'optimisation des tournées et de la flotte, équipent les camions de systèmes embarqués et recrutent des spécialistes de la logistique.

n effet, les TIC sont omniprésentes dans le transport et la logistique. Elles sont présentes ans les cabines via des technologies embarquées: on y trouve le plus souvent un

De son côté, le service « exploitation » des entreprises de transport se présente comme une salle de contrôle où les salariés, les yeux rivés sur leur écran, organisent l'activité. Il est d'ailleurs significatif, à cet égard, que l'on parle désormais davantage de prestation logistique et non plus seulement de transport.

Salle de contrôle 


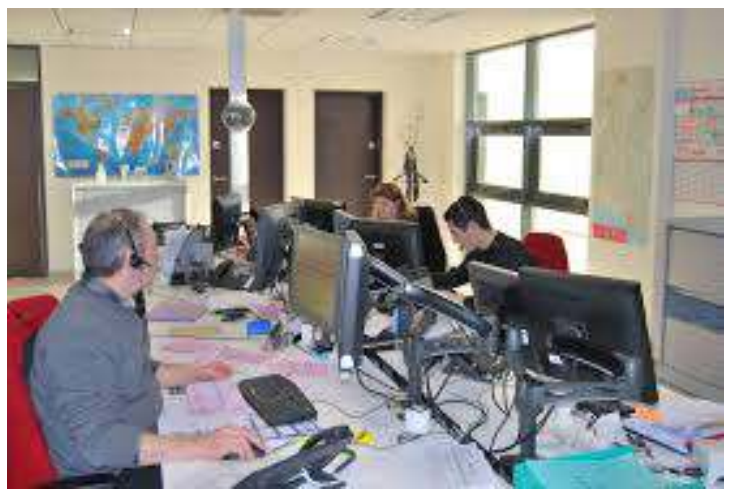

Ces investissements dans de nouveaux outils informatiques ont rationalisé l'organisation productive, et par conséquent celle du travail des salariés (conducteurs, agents de quai et exploitants). Ainsi, si l'organisation de la production a pu dépendre quasi exclusivement $\mathrm{du}$ " chauffeur routier ", elle relève dorénavant d'un service spécifique - exploitation ou logistique - pour satisfaire aux exigences de délais, de fiabilité et de coûts. En chassant les spécificités d'une culture de métier qui donnait au chauffeur une grande autonomie disparaît le mythe $d u$ " grand routier ». réduction continue de la taille du lot moyen, souvent associé à une augmentation de la charge moyenne transportée.

« Avant, avec Cassegrain, on chargeait 300 kilos pour Cassegrain! Aujourd'hui, on charge 25 tonnes de produits différents chez le même producteur ! Car le producteur, au lieu de remplir plein de petites palettes dans plein de petits camions, qui vont aller à chaque fois faire tous les magasins, il va remplir une semi ; cette semi, elle va aller sur une plateforme d'un grand distributeur, type Auchan, Carrefour, qui va dégrouper et stocker par catégorie » (un directeur).

22 Ce phénomène dit «d'allotissement » est une traduction de la complexification des flux de marchandises permise par les systèmes d'information et du grossissement des charges transportées.

« L'expéditeur charge une semi complète, mais il va avoir 25 points de destination tous programmés par l'informatique de l'acheteur qui va lui dire : "Vous me préparez autant de petites commandes que j'ai de magasins à aller livrer, mais vous me les mettez toutes dans le même camion" » (un directeur).

(a charge moyenne transportée et l'allotissement vont de pair avec l'augmentation du nombre de plates-formes. Sur ces plates-formes, les agents de quai déchargent des semi avec des marchandises pour de nombreuses destinations différentes et chargent d'autres semi pour une destination, mais remplies de marchandises issues de provenances différentes.

Des entreprises rencontrées, bon nombre évoquent également un processus de rétraction de leur espace d'intervention sur des pôles géographiques plus limités. Les réglementations en matière de temps de travail font que les conducteurs ont des obligations de coupure et de repos, alors que la marchandise doit être prise en charge le plus rapidement possible. Cette dissociation s'est faite en divisant un trajet en plusieurs et en affectant un véhicule à plusieurs conducteurs, d'où une réduction des distances parcourues par les conducteurs. 

la constitution d'une société disciplinaire et d'une société de contrôle.

\section{Temporalités et numériques}

\section{Connexion subie}

31 L'organisation du fret relève des salles d'exploitation qui constituent dorénavant le " poumon » des entreprises de TRM, car elles ont en charge l'organisation et le suivi des tournées. Les exploitants ${ }^{4} \mathrm{y}$ visualisent sur leur écran, via des cartes, où se situe la flotte de camions, et pour chacun d'entre eux, l'activité en cours : travail, conduite ou repos. Les tournées sont élaborées en combinant les demandes directes des clients, celles provenant des sites Internet de bourse de fret et les informations produites en temps réels par les différents dispositifs numériques qui équipent les cabines $\mathrm{du}$ camionchronotachygraphe, ordinateur de bord, GPS, téléphones portables.

Concernant les effets humains de la connexion via des équipements mobiles de télécommunication au sein des entreprises, on observe que les conducteurs routiers sont comme sur une chaîne de fabrication, c'est-à-dire que la hiérarchie peut désormais vérifier son planning, sa rentabilité, son efficacité. Il y a, en quelque sorte, "hertziennisation" du contrôle hiérarchique (Jauréguiberry, 2003).

«[...] Ils savent la route que tu as faite avant, par quel chemin tu es passé, et ils te localisent à une rue près, au numéro de rue près » (conducteur M., PL).

De façon générale, l'autonomie des employés nomades devait beaucoup à la disjonction entre leur temps et leurs espaces de travail. Parce qu'éloignés physiquement de tout contrôle, ils pouvaient se livrer à des "aménagements personnels » de leur temps de travail, pourvu que les tâches attendues soient effectuées. Pour les conducteurs routiers, cette évolution s'est faite au détriment d'habitudes de sociabilité et d'autonomie (Desfontaines, 2005). Par exemple, les choix d'itinéraires, des moments et de durées de conduite qui formaient les prérogatives du travail des conducteurs routiers leur échappent désormais. 
«Les anciens ne s'y retrouvent plus. Quand ils ont vu débarquer ces outils, ils se sont sentis traqués. Ils ont eu le sentiment de passer d'un régime de liberté totale à un régime de liberté surveillée. Quelque chose s'est cassée pour cette population quand ces outils ont été mis en œuvre » (un directeur). Néanmoins, il s'avère que c'est bien le côté subi de la connexion à un dispositif dont les traces numériques peuvent à tout moment produire des preuves qui opère comme un dispositif de contrôle. Car il existe, paradoxalement, une connexion choisie. En effet, tous les conducteurs routiers possèdent un téléphone portable privé en sus de celui fourni par l'entreprise, assorti, pour la plupart, d'un forfait illimité. Ils ont également, pour leur usage personnel, une télévision avec un lecteur de DVD et/ou un ordinateur avec des films téléchargés. Ils ont souvent un ordinateur connecté à Internet via la $3 \mathrm{G}$ et attendent avec impatience l'arrivée de la 4G.

Pour les conducteurs routiers, le téléphone remplace en partie la C.B., dont l'objectif est de lutter contre l'ennui au volant, la rengaine des kilomètres qui défilent, permettant autrement dit de «tuer le temps». Mais le téléphone portable privé permet aussi de renouer régulièrement avec la sphère privée.

«Le téléphone portable... avec la famille, les copains... Les week-ends sont très courts quand on part toute la semaine, on n'a pas forcément le temps de se voir. [...] On va s'accorder un peu plus de temps au téléphone, pour avoir l'impression de vivre un peu quelque chose quand même... » (conducteur M., PL).

« Des enfants? Oui, j'en ai trois. [...] On essaye une fois par jour de prendre des nouvelles. Là, ça va être l'école à nouveau, avoir des résultats, essayer que, malgré tout, elles aient le sentiment que le papa s'implique dans la vie de la famille, c'est ça quoi. [...] Et encore, moi je ne l'ai pas encore fait, mais c'est un investissement que je vais faire (tablette et $4 \mathrm{G}$ ), c'est aussi le fait d'avoir Skype, ça, c'est... je trouve que ça, c'est énorme! » (conducteur F., PL).

36 Ces pratiques participent d'une transformation sociale radicale liée à l'usage du téléphone portable, à savoir l'intégration en douceur de la sphère intime des employés dans leur sphère professionnelle (Broadbent, 2011). Le portable permet par exemple aux salariés de rester en contact tout au long de la journée avec leurs proches. Ces communications avec les êtres chers à partir du travail sont d'autant plus exacerbées par l'isolement et la solitude (ibid., p. 91).

Ces pratiques traduisent aussi une évolution du couple autonomie/contrôle toujours en tension. Si, dans un premier temps, la diminution d'autonomie de l'employé nomade réduit l'espace de liberté des employés qui leur est laissé pour gérer leur emploi du temps, elle permet à la direction de mieux contrôler ses employés distants afin de rendre leur travail plus efficace (Jauréguiberry, p. 445). Dorénavant, pour les employés nomades, isolés et connectés, l'autonomie renvoie à la possibilité de choisir avec qui et quand il se connecte avec ses proches et ce, tout au long de la journée de travail.

" Avec le forfait illimité, je peux rester 1 heure, 2 heures au téléphone, oui. C'est comme s'il était à côté de moi, et parfois on ne se parle pas pendant une demi-heure... » (conducteur B., PL). "Ça t'évite de t'endormir » (conducteur V., PL). "Ça m'arrive, quand j'appelle, je dis “bouge pas", je vais ouvrir les portes, le téléphone je le pose, et quand je reviens il est toujours là, des fois j'dis "bouge pas, j'vais vider", je sais qu'il est sur la route, je laisse le téléphone, le téléphone il peut être allumé pendant 3 
heures, je fais mes trucs, et puis voilà... Et puis des fois je prends le kit mains libres... Et pendant que je vide, je suis encore au téléphone... Ah ben, si on nous enlevait tout ! » (conducteur R., PL).

\section{Suivre le tempo : la discipline du flux}

Les TIC génèrent un rapport au temps inédit: si l'accélération des échanges accroît le sentiment et la demande d'autonomie, elle génère une dépendance liée au rythme qu'elle génère. Dans un premier temps, les responsables utilisaient les informations produites par les TIC embarquées pour recadrer les comportements et faire comprendre que, dès lors que la rémunération se fait au temps, que les durées de conduite sont contrôlées et que l'activité de transport est de plus en plus intégrée dans la chaîne logistique, c'est le respect des délais qui prévaut (Desfontaines, 2005). Depuis, les conducteurs constatent qu'il y a peu de surveillance proprement dite.

«[...] Ils ne s'en servent pas pour fliquer, des fois, ils ne savent même pas, eux, donc ils t'appellent : “T'es rendu où ?" "Ben, regarde sur la carte, tu verras bien." Ils ne s'en servent pas pour contrôler, car je me suis déjà trompé de route, j’ai fait des détours plusieurs fois et ils ne m'ont jamais rien dit » (conducteur R., PL).

Cependant, il s'avère que les traces laissées sont des preuves mobilisées en cas de désaccord et servent autant aux salariés qu'aux employeurs. Ainsi, les possibilités de contrôle permises par les dispositifs numériques orientent dorénavant les comportements des employeurs comme celui des conducteurs routiers.

L'optimisation des flux conduit également à organiser des relais de remorques qui passent d'un camion à un autre. Les conducteurs sont parfois informés en cours de route. Ils doivent ainsi se rendre disponibles à la logique du flux qui s'impose. Ce type de mobilisation des TIC permet d'expliquer une part des transformations du monde du travail, notamment le flux tendu qui engendre stress et sentiment d'insécurité permanente car le flux ne doit pas s'arrêter (Durand, 2004). Il s'agit bien là d'une discipline implicite qui impose la mobilisation permanente des salariés pour atteindre les objectifs productifs.

"Un autre inconvénient, c'était qu'un des aspects attractifs dans notre métier, c'était la liberté, ce qui voulait dire "je pars deux, trois ou cinq jours, et j'ai personne sur le dos". c'est de moins en moins vrai, car avec les déchargements fréquents et les flux tendus il faut être souvent en contact » (conducteur PL).

41 Autrement dit, le système d'information d'optimisation du fret, comme tout dispositif, est associé à une forme de pouvoir qui contribue au contrôle de l'activité des conducteurs et à la «fabrication des urgences ». Ce double contrôle, propre à l'hypermodernité, se caractérise par l'accélération continue des pratiques sociales (Carayol, 2005). En effet, la logique du flux et le nouveau "contrat social», celui qui rémunère au temps les conducteurs routiers, ont conduit ces derniers à abandonner l'étroite association qu'ils avaient construite entre travail et sociabilité (Desfontaines, 2005). Effectivement, les solidarités professionnelles s'estompent au profit du respect des délais :

«[...] Quand un gars tombait en panne au bord de la route... Oui, j'ai connu le début de la C.B., c'était ça. On tombait en panne, on s'arrêtait, enfin voilà... Il y avait ça, on prenait le temps, c'est fini, c'est un doux rêve aujourd'hui. [...] Et puis, si vous avez un pneu qui éclate, aujourd'hui vous avez une assistance, et globalement le collègue il ne va pas s'arrêter parce que le collègue il a un tempo derrière, parce qu'il doit livrer. Quand il va 
vous voir sur le bord de la route, ben il va filer en se disant que de toute façon il a le téléphone, chose qu'il n'avait pas avant, il y a les assistances, les machins et il va se débrouiller, donc voilà quoi... » (conducteur V., PL).

L'autonomie dont ils bénéficiaient auparavant en partant souvent loin et à la semaine était leur fierté. Dorénavant, il n'est quasiment plus possible pour les conducteurs de programmer un arrêt en fonction de leurs besoins physiologiques ou un détour pour retrouver amis ou collègues. Les conducteurs ont donc appris à ne plus prendre d'autres engagements que ceux que le travail impose et les restaurants routiers ont petit à petit disparu du paysage.

«Avec les collègues, ça devient plus compliqué... On se retrouvait à trois, quatre, on était sur les mêmes lignes, on se retrouvait aux mêmes endroits. Ça devient de plus en plus rare, de par cette flexibilité » (conducteur V., PL).

\section{Réflexivité et changement permanent}

Pour respecter la logique du flux et optimiser le temps, les entreprises organisent leur activité par "rendez-vous », organisation qui est devenue la norme dans le secteur. C'est pourquoi les conducteurs doivent impérativement informer des chargements/ déchargements. L'exploitant fournit alors la liste des frets suivants. Selon les entreprises, ces échanges se font via l'ordinateur de bord ou par téléphone. Les technologies embarquées permettent également aux exploitants d'ajouter des courses, de remanier des itinéraires, notamment en fonction des temps de service du conducteur et de sa proximité avec le nouveau lieu de chargement.

«[...] Ils ont toutes les heures de conduite et tout sur leurs ordinateurs, ils voient tout, ils savent où t'es rendu, pour eux, s'ils trouvent de la marchandise quelque part, voir si tu peux aller... Ça évite les coups de téléphone répétitifs, les machins, les calculs...» (conducteur M., PL).

Généralement, l'horizon temporel ne dépasse pas les 24 heures et s'obscurcit parfois jusqu'à faire attendre le chauffeur sur un parking pour connaître son prochain rendezvous.

« Lundi, je suis parti à 5 heures du dépôt, je savais tout ce que j'avais à faire dans la journée et le soir, quand j'ai appelé, ils m'ont donné toutes les ramasses que j'avais à faire le lendemain. [...] J’ai déchargé et rechargé au dépôt, et le lendemain, mardi, une fois vide, j'ai appelé, il était 11 heures et ils m'ont fait attendre jusqu'à 14 heures. C'est parce qu'il n'y avait pas de boulot, ils regardaient sur "bourse fret", ils sont sur les sites pour regarder qu'est-ce qu'il y a à aller chercher, le prix, combien... » (conducteur R., PL).

Les technologies de l'information et de la communication ont permis de résoudre bon nombre de problèmes soulevés par la complexité des tournées; elles ont aussi permis de capter et de produire de nouvelles informations et de les faire circuler de plus en plus vite. Ces informations permettent de développer des connaissances nouvelles sur les pratiques professionnelles et leurs organisations, qui conduisent alors à les revoir et à les améliorer dans un mouvement réflexif permanent. Ce mouvement passe par la mise en place de nouvelles formes de flexibilité en matière de ressources humaines. Ainsi, l'optimisation des camions exige également des horaires variables et des tournées plus courtes. 
« Il faudrait plus de régularités sur les tournées. En couple, c'est difficile. Le nombre de divorces est impressionnant chez les conducteurs » (conducteur S., VL).

«La difficulté, c'est les horaires, surtout le samedi : $13 \mathrm{~h}-21 \mathrm{~h}$. On n'a pas de vie sociale (agent de quai).

De leur côté, les salariés, qu'ils soient conducteurs ou agents de quai, vont plutôt évoquer en premier lieu les difficultés de récupération en matière de sommeil en raison des horaires de travail et les risques que cela entraîne pour la conduite, la manutention, etc. « Beaucoup ont du mal à récupérer, surtout ceux qui n'arrivent pas à dormir n'importe quand. Les anciens ont un ras-le-bol de ce rythme » (conducteur R., PL).

\section{Conclusion}

47 A partir d'une recherche sur l'évolution des organisations et des métiers dans le secteur du TRM, nous avons interrogé l'articulation entre les modalités de contrôle et la dimension temporelle. Si la théorie économique néoclassique n'a pas été remise en cause alors qu'elle cantonnait le travail à sa force produite et au temps écoulé, c'est parce que cette représentation abstraite du travail a permis la mise en place d'une société disciplinaire et d'une société de contrôle.

En effet, le diktat du délai à respecter, la discipline du flux, l'impératif de l'urgence opèrent comme une force supérieure qui guide les comportements au travail.

Ce modèle disciplinaire imposé par les délais ne correspond pas exactement au modèle théorisé par Foucault. Pour ce dernier, les sociétés disciplinaires ont besoin d'un monde clos, d'enfermement, où le regard est omniscient. Cependant, Foucault pressent la fin de ce modèle et l'émergence une autre forme de pouvoir, qui s'inscrit dans l'individualisation de nos sociétés et la fin de ces mondes clos. Effectivement, pour Deleuze, qui a poursuivi cette réflexion, ce modèle est en crise pour laisser la place à une " société de contrôle ». Celle-ci se caractérise par l'émergence de nouvelles organisations structurées par les TIC, qui permettent de :

"garantir une plus grande marge de manœuvre aux individus, des espaces-temps plus ouverts et flexibles, davantage de mobilité, mais en apparence seulement. Car, contrairement aux dispositifs disciplinaires, qui procèdent par la coercition et la concentration des corps, le mouvement et la liberté de circulation sont les conditions nécessaires à l'exercice d'un pouvoir qui opère désormais par « contrôle continu » de tous les aspects de l'existence et par « communication instantanée » (Pironet, 2007, p.15).

Ainsi, les dispositifs informationnels embarqués dans les camions renvoient à cette "société de contrôle» (Wikipédia, 2016). Cependant, nous considérons que le TRM, via des rappels permanents des délais à respecter, agit de même que les sociétés disciplinaires qui cherchent à conformer les corps aux comportements attendus. Mais également selon les modalités d'une société de contrôle avancée par Deleuze où les modalités de l'application d'une contrainte sur un corps changent : on passe à des effets incitateurs. Cette société de contrôle opère également par atomisation des individus qui ne peuvent plus s'organiser pour se retrouver. De plus, l'équipement numérique privé embarqué dans les camions (smartphone, ordinateur, téléviseur, etc.) permet de supporter cette solitude en se divertissant et de réactiver les liens affectifs et amicaux.

51 En jouant ainsi sur les différentes sphères (professionnelles, privées, publiques), les usages du numérique sont bien au cœur du couple autonomie/contrôle. 
ANDONOVA Y. et VACHER B., 2013, « Nouvelles Formes de visibilité des individus en entreprise : technologie et temporalité », Communication \& Organisation, $\mathrm{n}^{\circ} 44$, Presses universitaires de Bordeaux, pp. 5-14.

AUBERT N., 2009, Le Culte de l'urgence : La Société malade du temps, Paris, Flammarion, coll. «Champs Essais ».

AUBERT N., 2006, «L'Urgence, symptôme de l'hypermodernité : de la quête de sens à la recherche de sensations ", Communication \& Organisation, $n^{\circ} 29$, Presses universitaires de Bordeaux, pp. $11-21$.

BERNARDET M., 1999, Le Transport routier de marchandise : Fonctionnements et dysfonctionnements, Paris, Economica.

BOUTON C., 2013, Le Temps de l'urgence, Lormont, Éditions Le Bord de l'eau, coll. « Diagnostics ». BROADBENT S., 2011, L'Intimité au travail, Limoges, FYP Éditions.

CARAYOL V., 2005, « Principe de contrôle, communication et temporalités organisationnelles », Études de communication, $\mathrm{n}^{\circ} 28$, pp. 77-89.

CEP Transports, 2013, Céreq, Cope 13, Netstear, EMN, AFT-IFTIM (dir.), Opca Transports et Services, DGEFP.

DESFONTAINES H., 2005, « Apprentissage des normes temporelles du travail salarié, un nouveau défi pour les chauffeurs routiers » in D. Linhart et A. Moutet (dir.), Le travail nous est compté : La Construction des normes temporelles du travail, Paris, La Découverte, pp. 190-214.

DESFONTAINES H., 2005, « Le Travail des chauffeurs routiers de marchandises », Travail et Emploi, $\mathrm{n}^{\circ}$ 104, pp. 29-42.

DURAND J.-P., 2012, La Chaîne invisible. Travailler aujourd'hui : flux tendu et servitude volontaire, Paris, Le Seuil, coll. « Économie humaine ».

GHEORGHIU M. D. et MOATTY F., 2005, « Groupes sociaux et enjeux de la coopération au travail dans l'industrie », Réseaux, n 134, pp. 91-122.

GOLLAC M., MANGEMATIN V., MOATTY F. et SAINT-LAURENT A.-F. (de), 1999, « À quoi sert donc l'informatique? Revue d'études de cas », in Innovations et performances : approches interdisciplinaires , sous la direction de D. FORAY et J. MAIRESSE, Paris, Éditions de l'EHESS.

JAURÉGUIBERRY F., 2003, « La Mise en place des télécommunications mobiles au sein des entreprises comme vecteur de nouvelles formes d'exploitation », in Actes des neuvièmes journées de sociologie du travail : Contraintes, normes et compétences au travail, Paris, université de Paris X, tome 1 , pp. 443-451.

KLEIN T. et RATIER D., 2012, L'Impact des TIC sur les conditions de travail, Centre d'analyse stratégique, DGT, Paris, La Documentation française, coll. « Rapports \& documents ».

KOGAN A.-F., (2009), « La Mise en jeu du corps dans l'activité de téléphonie », Études de communication, langages, information, médiations, $\mathrm{n}^{\circ} 33$, pp. 171-186.

SAINT-LAURENT-KOGAN A.-F. (de), 2013, « Le Travail à l'heure du numérique », Cahiers français, n - 372 : «La Société numérique », Paris, La Documentation française, pp. 27-32.

Wikipédia (2016), « Société de contrôle », in Wikipédia, l'encyclopédie libre, https:// fr.wikipedia.org/wiki/Société_de_contrôle, dernière consultation le 8 octobre 2016.

PIRONET O., (2007), «Gilles Deleuze (1925-1995). Le théoricien des sociétés de contrôle », Manière de voir, $\mathrm{n}^{\circ}$ 96, décembre 2007-janvier 2008. 
VENDRAMIN P., 2006, « Les TIC, complices de l'intensification du travail », in Organisation et intensité du travail, sous la direction de P. Askenazy, D. Cartron, F. De Coninck et M. Gollac, Toulouse, Octares Éditions, pp. 129-135, coll. « Le travail en débats ».

VIRILIO P., 1995, La Vitesse de libération, Paris, Éditions Galilée.

\section{NOTES}

1. CEP : Contrat d'Études Prospectives. Ce CEP Transports a été réalisé par un consortium à la demande de la DGEFP et de l'Opca Transports et Services.

2. Le terme officiel est conducteur VL (véhicule léger) ou PL (poids lourd), mais l'expression « chauffeur routier » reste encore largement utilisée.

3. Source : DAEI/SES Insee, enquête TRM 2003.

4. Dans le TRM, les exploitants, généralement de formation bac +2 , ont pour mission d'organiser les tournées en optimisant la flotte. Ils sont en contact avec les clients et les conducteurs.

\section{ABSTRACTS}

This paper is based on a research carried on road freight transport. It questions particularly the different links between modalities of control and the organization of time which is central to freighting. These organizations operate in a capitalist system where work is defined exclusively in terms of productive labour and time spent. This "abstract" representation of work encourages the implementation of a disciplinary society (disciplining the flow) and of a control society (the proliferation of digital footprints which induces behaviours), from the perspective of Foucault and Deleuze.

Cet article s'appuie sur une recherche sur l'évolution des organisations et des métiers dans le secteur du TRM (transport routier de marchandises). Il présente les différentes articulations entre les modalités de contrôle et la dimension temporelle qui est au cœur de l'organisation du fret. Ces organisations évoluent dans un système capitaliste où le travail n'est considéré que par la force produite et le temps écoulé. Cette représentation « abstraite » du travail permet la mise en place d'une société disciplinaire (la discipline du flux auquel on se soumet) et d'une société de contrôle (la prolifération des traces numériques a des effets incitateurs sur les comportements), dans la perspective ouverte par Foucault et Deleuze.

Este artículo está basado en una investigación sobre la evolución de las organizaciones y de los oficios en el sector del TCM (transporte por carretera de mercancías). Muestra las distintas relaciones entre las modalidades de control y la dimensión temporal que se sitúa en el centro de la organización de las mercancías transportadas. Las organizaciones evolucionan en un sistema capitalista, en donde el trabajo únicamente es considerado por la fuerza producida y por el 
tiempo utilizado. Desde la perspectiva establecida por Foucault y Deleuze, puede señalarse que esta representación "abstracta" del trabajo permite la instauración de una sociedad disciplinaria (la disciplina del flujo al cual se somete) a la vez que de una sociedad de control (la proliferación de los rastros digitales tiene efectos instigadores sobre los comportamientos).

\section{INDEX}

Mots-clés: fret, numérique, organisation, temps, contrôle

Keywords: road freight, transportation, organization, time, control

Palabras claves: flete, digital, organización, tiempo, control.

\section{AUTHOR}

\section{ANNE-FRANCE KOGAN}

Anne-France Kogan est Professeure en sciences de l'information et de la communication à l'Université de Rennes 2 et chercheure au PREFics, Ses enseignement s'inscrivent dans la thématique « Société et numérique ». Ses recherches portent sur les modalités de diffusion du numérique et les transformations engendrées par celui-ci sur le travail, les métiers et les organisations. 